\title{
One and Two-Dimensional Pattern Formation on Ion Sputtered Silicon
}

\section{Citation}

Brown, Ari-David, H. Bola George, Michael J. Aziz, and Jonah Erlebacher. 2004. One and TwoDimensional Pattern Formation on Ion Sputtered Silicon. Materials Research Society Symposium Proceedings 792, R7.8.

\section{Published Version}

http://72.22.18.215/s_mrs/sec_subscribe.asp?CID=2633\&DID=116922\&action=detail

\section{Permanent link}

http://nrs.harvard.edu/urn-3:HUL.InstRepos:2794948

\section{Terms of Use}

This article was downloaded from Harvard University's DASH repository, and is made available under the terms and conditions applicable to Other Posted Material, as set forth at http:// nrs.harvard.edu/urn-3:HUL.InstRepos:dash.current.terms-of-use\#LAA

\section{Share Your Story}

The Harvard community has made this article openly available.

Please share how this access benefits you. Submit a story.

\section{Accessibility}




\title{
One and Two-Dimensional Pattern Formation on Ion Sputtered Silicon
}

\author{
Ari-David Brown $^{1,2}$, H. Bola George ${ }^{3}$, Michael J. Aziz ${ }^{3}$, and Jonah Erlebacher ${ }^{2}$ \\ ${ }^{1}$ Department of Physics and Astronomy, Johns Hopkins University, \\ Baltimore, MD 21218, U.S.A. \\ ${ }^{2}$ Department of Materials Science and Engineering, Johns Hopkins University, \\ Baltimore, MD 21218, U.S.A. \\ ${ }^{3}$ Division of Engineering and Applied Sciences, Harvard University, \\ Cambridge, MA 02138, U.S.A.
}

\begin{abstract}
The evolution of surface morphology during ion beam erosion of $\mathrm{Si}(111)$ at glancing ion incidence $\left(60^{\circ}\right.$ from normal, $500 \mathrm{eV} \mathrm{Ar}^{+}, 0.75 \mathrm{~mA} / \mathrm{cm}^{2}$ collimated beam current) was studied over a temperature range of $500-730^{\circ}$ Celsius. Keeping ion flux, incident angle, and energy fixed, it was found that one-dimensional sputter ripples with wavevector oriented perpendicular to the projected ion beam direction form during sputtering at the lower end of the temperature range. For temperatures above approximately $690^{\circ}$ Celsius, growth modes both parallel and perpendicular to the projected ion beam direction contribute to the surface morphological evolution. This effect leads to the formation of bumps ("dots") with nearly rectangular symmetry.
\end{abstract}

\section{INTRODUCTION}

Sputter rippling is a term commonly used to describe the self-assembly of periodic nanostructures on surfaces etched by an ion beam. The surface instability leading to rippling is caused by a competition between ion-induced roughening on one hand, and smoothing due to surface relaxation mechanisms such as surface diffusion on the other. Ripples have been produced on the surfaces of a variety of different materials including metals [1, 2], semiconductors [3], and amorphous solids [4, 5]. In addition, many different ion species and ion energies have been used $[3,6]$ to form sputter ripples.

One- and two-dimensional features with well-defined symmetry have been produced by sputter rippling. Arrays consisting of small bumps or "nano-dots" with hexagonal symmetry have been fabricated on GaSb [7] and $\mathrm{Si}$ [8] surfaces using normal incidence sputtering; similar structures were formed on InP [9] during simultaneous ion bombardment and sample rotation. Typically, only one-dimensional features form during glancing incidence sputtering, and typically two-dimensional features tend to exhibit only hexagonal ordering. Here we report the evidence of two-dimensional pattern formation with nearly rectangular symmetry on $\mathrm{Si}(111)$ using glancing incidence sputtering. In fact, we find that different sputter ripple morphologies on $\mathrm{Si}(111)$ may be generated simply by varying the substrate temperature.

A theory explaining the morphological evolution of ion sputtered surfaces was formulated by Bradley and Harper (BH) [10]. BH use a curvature-dependent sputter yield derived from Sigmund's model for ion sputtering [11] and couple this ion-induced roughening mechanism to surface smoothing by surface diffusion. The cooperative 
behavior between roughening and smoothing mechanisms leads to functional relationships between ripple wavelength, ion flux and substrate temperature. Modifications to the theory account for additional smoothing mechanisms such as ion-induced effective surface diffusion [12], ion-enhanced viscous flow (IVF) [13], and ion-induced momentum transfer [14].

$\mathrm{BH}$ theory predicts a ripple amplification factor, $R_{q}$, which controls the growth rate as a function of time $t$ of each Fourier component of the surface morphology. Specifically, if we let $h_{q}$ be the amplitude of the Fourier component with wavelength $\lambda=2 \pi q$, then $h_{q}(t)$ evolves according to

$$
h_{q}(t)=h_{q}(0) e^{R_{q} t}
$$

The amplification factor $R_{q}$ is related to the microscopic mechanisms of ion-induced roughening and smoothing by surface diffusion by

$$
R_{q}=-f a \Omega Y_{0}(\theta) \Gamma(\theta) q^{2}-\frac{D_{s} C \gamma \Omega^{2}}{k_{B} T} q^{4}
$$

Here, $f$ is the ion flux, $a$ is the ion range, $\Omega$ is the atomic volume, $Y_{0}(\theta)$ is the sputter yield of a flat surface, $\theta$ is the angle between the ion beam and the surface normal, $\Gamma(\theta)$ is a negative quantity that accounts for the curvature-dependence of the yield, $D_{s}$ is the surface diffusivity, $C$ is the concentration of mobile species participating in surface diffusion, $\gamma$ is the surface energy, $k_{B}$ is Boltzmann's constant, and $T$ is the substrate temperature. The observed ripple wavelength is equal to the fastest growing wavelength $\lambda_{\max }$ and is given by:

$$
\lambda_{\max }=2 \pi\left(\frac{2 D_{s} C \gamma \Omega}{f a Y_{0}(\theta)|\Gamma(\theta)| k_{B} T}\right)^{1 / 2} \sim T^{-1 / 2} e^{-E / 2 k_{B} T},
$$

where $E$ is the activation energy for surface diffusion, and the primary functional dependence of $\lambda_{\max }$ on temperature is indicated.

\section{EXPERIMENTAL DETAILS}

For all results presented here, ripples were produced on 3" Si(111) wafers (n-type, $\rho=$ $0.002-0.005 \Omega \mathrm{cm}$ ) inside an UHV chamber with a base pressure of $6.0 \times 10^{-11}$ Torr. Sputter ripples were produced using a $3 \mathrm{~cm}$ collimated Ion Tech (Fort Collins, Colorado) ion source that generated an ion beam consisting of $500 \mathrm{eV} \mathrm{Ar}^{+}$ions at a flux of $0.75 \mathrm{~mA} / \mathrm{cm}^{2}$, directed $60^{\circ}$ from normal incidence. The projected beam was parallel to the [1 $\left.\overline{1} 0\right]$ direction on the Si target. Sample temperature was controlled using a pyrolitic boron nitride graphite filament heater and was monitored using a thermocouple and an optical pyrometer.

The time evolution of sputter ripple morphology was investigated using in situ UV light scattering spectroscopy (LiSSp) and atomic force microscopy (AFM). LiSSp operates by 
measuring non-specular scattered light from an illuminated region on the sample at a fixed detection angle, and provides a real-time measurement of the power spectral density of the surface morphology [15]. A $300 \mathrm{~W}$ broadband Xe lamp was used for incident light illumination. In our experimental geometry, LiSSp is able to probe ripple periodicities between $450 \mathrm{~nm}$ and $1.1 \mu \mathrm{m}$. LiSSp spectra were taken at different time intervals during the course of the ripple growth runs. As the morphological Fourier components of a sputter ripple started to dominate the surface topography, the intensity of the LiSSp-obtained surface power spectrum increased, peaking at a wavelength correlated to the ripple wavelength. As discussed below, during this "early time" regime each Fourier component of the ripple morphology grows exponentially fast with time. After long sputtering times, we observe ripple amplitude saturation and random surface roughening, similar to observations made on sputter ripples on $\mathrm{Si}(001)$ [16]. To avoid complications associated with non-linear morphological evolution in the "long time" regime, all reported wavelength and morphological data were obtained prior to the time at which the characteristic ripple amplitudes first reached their maximal values. Ex situ AFM using a Digital Instruments (Woodbury, New York) Nanoscope III was used to confirm the wavelength and morphology information obtained by LiSSp and also allowed for the observation of ripples with wavelengths outside of the LiSSp detection range.

\section{RESULTS AND DISCUSSION}

\section{Observed ripple morphologies}

An AFM micrograph of ripples produced at $607^{\circ} \mathrm{C}$ is shown in Figure 1. These ripples have a wavelength of approximately $475 \mathrm{~nm}$ and mean amplitude of $15 \mathrm{~nm}$. In this case, the corrugation direction is parallel to the projected ion beam direction, i.e., the wavevector of the surface ripple morphology is perpendicular to the projected ion beam direction. Using reasonable estimates for the input parameters to $\mathrm{BH}$ theory, such a morphology is indeed predicted by $\mathrm{BH}$ theory for $\theta=60^{\circ}$.

As the sample temperature is increased, we have found the surprising result that the ripple morphology transforms into a morphology consisting of a two-dimensional array of bumps (dots) exhibiting nearly rectangular symmetry. Figure 2 shows a representative AFM micrograph of such a morphology, illustrating two-dimensional ripples formed after $577 \mathrm{~min}$ of sputtering at $717^{\circ} \mathrm{C}$. The wavelength of the features parallel to the projected beam (i.e., the orientation at lower temperatures) is roughly $1.4 \mu \mathrm{m}$; those perpendicular to the beam have periodicities of around $650 \mathrm{~nm}$. The dot amplitudes are approximately $5 \mathrm{~nm}$.

\section{Temperature dependence of the ripple wavelength}

One-dimensional ripple evolution at temperatures below approximately $690{ }^{\circ} \mathrm{C}$ is welldescribed by the $\mathrm{BH}$ model. Specifically, a unique, time-independent ripple wavelength could be identified, and the amplitude of this ripple grew exponentially fast at early times. It was also found that the ripple wavelength increased with temperature. LiSSp and AFM measurements of the wavelength variation versus temperature are presented in Figure 3 (squares and circles, respectively). For temperatures below $\sim 690{ }^{\circ} \mathrm{C}$, the data are well fit by a function of the form $\lambda \sim \mathrm{T}^{-0.5} \mathrm{e}^{-\mathrm{A} / \mathrm{T}}$, where $A$ is a constant. Fitting to $\mathrm{BH}$ theory through Eq. 
(3) yields an activation energy for surface relaxation of $1.0+/-0.1 \mathrm{eV}$. This value agrees with other measurements of the activation energy for surface self-diffusion on $\mathrm{Si}(111)$ measured using different techniques [17]. Thus, the sputter rippling behavior is consistent with surface diffusion being the primary smoothing mechanism during ion sputtering from $500^{\circ} \mathrm{C}$ to $\sim 690^{\circ} \mathrm{C}$.

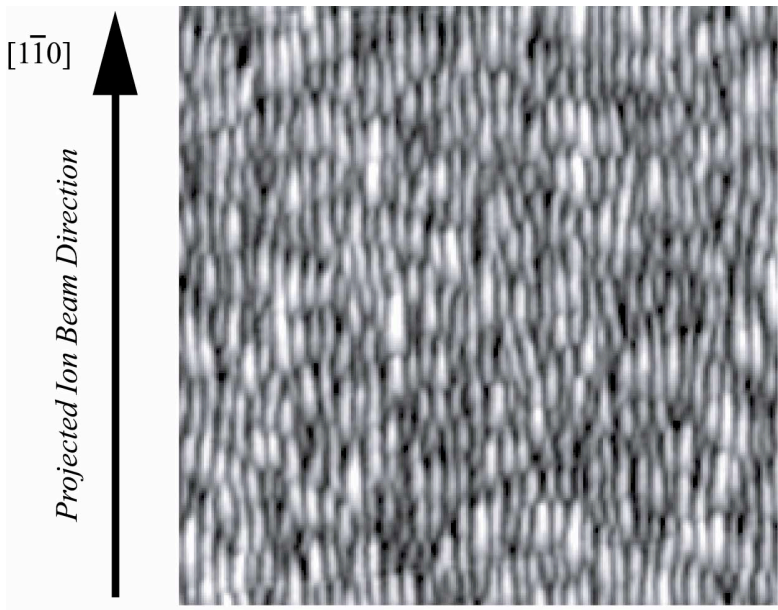

$2 \mu \mathrm{m}$

Figure 1. Sputter rippled $\mathrm{Si}(111)$, showing one-dimensional ripple morphology. Sample temperature $=607{ }^{\circ} \mathrm{C}$. The ion flux $f=0.75 \mathrm{~mA} / \mathrm{cm}^{2}$; ion fluence $=2.1 \times 10^{19} \mathrm{~cm}^{-2}$. Ion beam incidence angle $\theta=60^{\circ}$ from normal. Ripple wavelength $=475 \mathrm{~nm}$; ripple amplitude $=15 \mathrm{~nm}$.

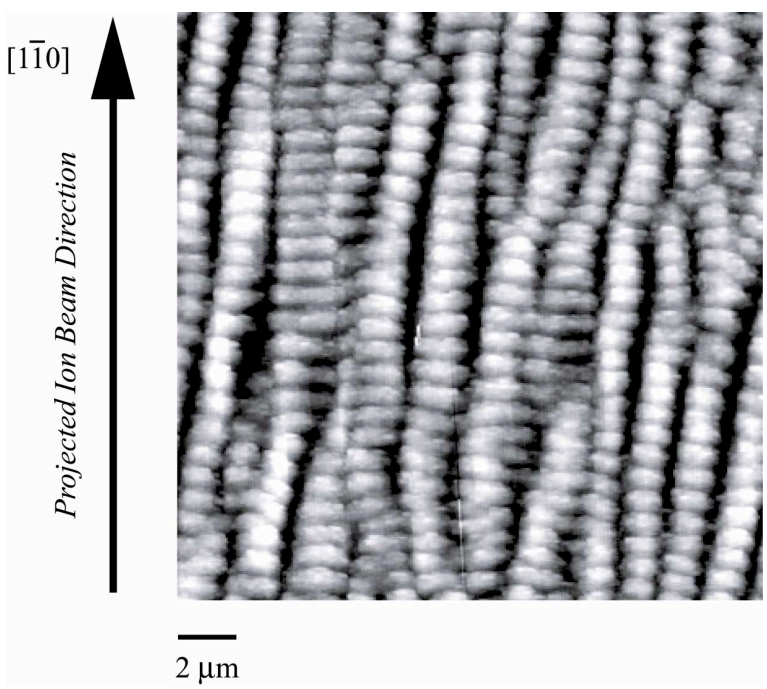

Figure 2. Self-organized two-dimensional array of ion sputter dots sputtered by $500 \mathrm{eV} \mathrm{Ar}^{+}$ ions at $\theta=60^{\circ}$ from normal. $T=717{ }^{\circ} \mathrm{C} ; f=0.75 \mathrm{~mA} / \mathrm{cm}^{2}$; ion fluence $=1.6 \times 10^{20} \mathrm{~cm}^{-2}$. Dot dimensions are approximately $1400 \mathrm{~nm}$ x $650 \mathrm{~nm}$. Dot amplitude $=5 \mathrm{~nm}$. 
During sputtering at temperatures above $\sim 690{ }^{\circ} \mathrm{C}-730{ }^{\circ} \mathrm{C}$, we observed differences in the way the surface morphology evolves as compared to the lower-temperature ripples. These differences were manifested as follows:

- The ripple morphology exhibited two-dimensional periodicity.

- For constant flux, the aspect ratio of the two-dimensional ripples was independent of substrate temperature within our measured temperature range.

- The time evolution of two-dimensional ripples followed the trend that at early times ripples with wavevector parallel to the projected ion beam direction formed (i.e., opposite to the low-temperature orientation); only at later times longer-wavelength features with wavevector perpendicular to the projected ion beam direction grow to the same amplitude. The combination of these two mutually perpendicular growth modes leads to the two-dimensional periodicity.

Our results are a significant deviation from $\mathrm{BH}$ behavior, which predicts that only one orientation of ripple morphology should be observed for fixed ion flux and incidence angle. The extent of this deviation may be assessed by noting (see Figure 3), that the wavelength of the corrugation in the direction perpendicular to the projected ion beam sharply deviates from the $\mathrm{BH}$ trend starting at $\sim 690{ }^{\circ} \mathrm{C}$, and that this feature size remains more-or-less temperature independent over the temperature region probed. Similar temperature independence was observed for ripples oriented perpendicular to the beam direction. These results imply that other surface relaxation mechanisms may be operative. Both ion-induced viscous flow [13] and ion-induced effective surface diffusion [12] are predicted to yield temperature-independent surface morphologies at low temperature; however, these relaxation mechanisms are overwhelmed at higher temperature by thermally activated mechanisms that result in a ripple wavelength increasing with temperature. Additional studies incorporating the wavelength dependence of ion flux and ion energy are ongoing in order to determine the nature of the two-dimensional surface morphology evolution.

Figure 3. Wavelengths of sputter ripple features with wavevector oriented perpendicular to projected ion beam versus sample temperature. Measurements were obtained using LiSSp (squares) and AFM (dots). One-dimensional ripples are observed below $\sim 690{ }^{\circ} \mathrm{C}$, whereas two-dimensional ripples are observed above this temperature. A fit of the one-dimensional ripples to the wavelength behavior predicted by BradleyHarper theory, Eq. (3), is shown as a solid

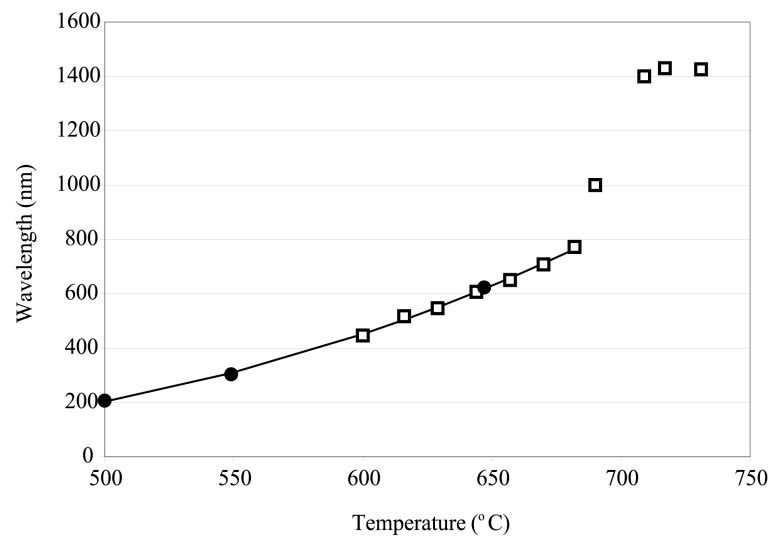
line.

\section{CONCLUSIONS}

Glancing incidence ion bombardment has been shown to produce one and twodimensional sputter ripple morphologies on sputtered $\mathrm{Si}(111)$. For temperatures below 
$\sim 690{ }^{\circ} \mathrm{C}$ at an ion flux of $0.75 \mathrm{~mA} / \mathrm{cm}^{2}$, ripples oriented with wavevectors perpendicular to the projected ion beam are formed, consistent with Bradley-Harper theory incorporating ioninduced roughening in competition with surface relaxation due to surface diffusion. The activation energy for surface diffusion was extracted from the ripple wavelength temperature dependence and found to equal $1.0+/-0.1 \mathrm{eV}$. The two-dimensional morphology observed when sputtering samples at temperatures above $\sim 690^{\circ} \mathrm{C}$ has not been seen previously in sputter rippling experiments. This novel structure further expands the variety of features that may be formed by sputter rippling.

\section{ACKNOWLEDGMENTS}

We gratefully acknowledge useful discussions with Y. Ding and A. Mathur. We also thank K. Stebe, N. Pesika, and F. Feng for their generous assistance with AFM. Work at Johns Hopkins and Harvard are supported by the United States Department of Energy, Basic Energy Sciences under award no. DE-FG02-01ER45942 and DE-FG02-01ER45947, respectively.

\section{REFERENCES}

1. F. Vasiliu, I. A. Teodorescu, and F. Glodeanu, J. Mater. Sci. 10, 399 (1975).

2. S. Rusponi, G. Constantini, C. Boragno, and U. Valbusa, Phys. Rev. Lett. 814184 (1998).

3. J. Erlebacher, M. J. Aziz, E. Chason, M.B. Sinclair, J. A. Floro, Phys. Rev. Lett. 82, 2330 (1999).

4. M. Navez, C. Sella, and D. Chaperot, C. R. Acad. Sci. 254, 240 (1962).

5. E. Chason, T. M. Mayer, B. K. Kellerman, D. T. McIlroy, and A. J. Howard, Phys. Rev. Lett. 72, 3040 (1994).

6. T.K. Chini, F. Okuyama, M. Tanemura, and K. Nordlund, Phys. Rev. B 67, 205403 (2003).

7. S. Facsko, T. Dekorsy, C. Koerdt, C. Trappe, H. Kurz, A. Vogt, and H. L. Hartnagel, Science 285, 1551 (1999).

8. R. Gago, L. Vazquez, R. Cuerno, M. Varela, C. Ballesteros, J. M. Albella, Appl. Phys. Lett. 78, 3316 (2001).

9. F. Frost, A. Schindler, and F. Bigl, Phys. Rev. Lett., 85, 4116 (2000).

10. R. M. Bradley and J. M. E. Harper, J. Vac. Sci. Technol. A 6, 2390 (1988).

11. P. Sigmund, J. Mater. Sci. 8, 1545 (1973).

12. M. A. Makeev and A. -L. Barabasi, Appl. Phys. Lett. 71, 2800 (1997).

13. C. C. Umbach, R. L. Headrick, and K. -C. Chang, Phys. Rev. Lett. 87, 246104 (2001).

14. G. Carter and V. Vishnyakov, Phys. Rev. B 54, 17647 (1996).

15. E. Chason, M. B. Sinclair, J. A. Floro, J. A. Hunter, and R. Q. Hwang, Appl. Phys. Lett, 72, 3276 (1997).

16. J. Erlebacher, M. J. Aziz, E. Chason, M. B. Sinclair, and J. A. Floro, J. Vac. Sci. Tech. A 18, 115 (2000).

17. H. -C. Jeong and E. D. Williams, Surf. Sci. Rep. 34, 171 (1999). 